CIC. Cuadernos de Información y Comunicación

ISSN: 1135-7991

http://dx.doi.org/10.5209/ciyc.68743

\title{
La contribución de Wenceslao Castañares a los estudios de C. S. Peirce
}

\author{
Sara Barrena y Jaime Nubiola ${ }^{1}$
}

Enviado: 10/04/2020 / Aceptado: 16/04/2020

Resumen. Este texto describe la producción científica del profesor Wenceslao Castañares incidiendo en su contribución al conocimiento de la obra de Charles Sanders Peirce. Wenceslao Castañares -por delante y por encima de cualquier otro- fue la persona clave para el descubrimiento del pensamiento de Peirce en el mundo hispánico. No solo logró que se le llegara a conocer, sino que contribuyó decisivamente a la comprensión de la obra peirceana y al desarrollo de las cuestiones que el pensamiento de Peirce planteaba en muy diversas áreas. Los autores revisan el perfil humano, las aportaciones esenciales y valoran en detalle la importancia del legado de este profesor.

Palabras clave: Wenceslao Castañares; C.S; Peirce; semiótica; obra perciana; estudios sobre Peirce; Historia del Pensamiento Semiótico.

\section{[en] The contribution of Wenceslao Castañares to the studies of C. S. Peirce}

\begin{abstract}
This text describes the scientific production of Professor Wenceslao Castañares, noting specifically his contribution to the knowledge of the works of Charles Sanders Peirce. Wenceslao Castañares -ahead and above anyone else- was the key person in discovering Peirce's thought in the Hispanic world. Not only did he get to know him, but he contributed decisively to the understanding of the Peircean works and to the development of the questions that Peirce's thought posed in many different areas. The authors review the human profile, the essential contributions and assess in detail the importance of this professor's legacy.

Keywords: Wenceslao Castañares; C.S; Peirce; Semiotics; Percian work; studies on Peirce; History of Semiotic Thought.
\end{abstract}

Sumario: 1. Breve semblanza personal de Wenceslao Castañares. 2. Principales escritos de Wenceslao Castañares en relación con C. S. Peirce. 3. La aportación de Wenceslao Castañares a los estudios peirceanos.

Cómo citar: Barrena, S.; Nubiola, J. (2020). La contribución de Wenceslao Castañares a los estudios de C. S. Peirce, en CIC. Cuadernos de Información y Comunicación 25, 23-30.

Charles S. Peirce (1839-1914), científico, lógico y filósofo norteamericano, ha sido considerado como fundador del pragmatismo y padre de la semiótica contemporánea

1 Los profesores Jaime Nubiola y Sara Barrena son investigadores del Grupo de Estudios Peirceanos en la Universidad de Navarra y los mayores especialistas en la obra de C.S. Peirce en nuestro país.

E-mail: sbarrena@unav.es

E-mail: jnubiola@unav.es 
entendida como teoría filosófica de la significación y de la representación. El pensamiento de Peirce constituye uno de las más ricos y profundos de los últimos siglos y ha adquirido gran relieve en las últimas décadas en diferentes campos de la cultura: lógica, filosofía, semiótica, astronomía, geodesia, matemáticas, teoría e historia de la ciencia, econometría y psicología.

Wenceslao Castañares — por delante y por encima de cualquier otro - fue la persona clave para el descubrimiento del pensamiento de Peirce en el mundo hispánico. No solo logró que se le llegara a conocer, sino que contribuyó decisivamente a la comprensión de la obra peirceana y al desarrollo de las cuestiones que el pensamiento de Peirce planteaba en muy diversas áreas. La profunda formación filosófica y la amplia erudición histórica de Wenceslao aportaron un excelente rigor académico y una solidez intelectual a los estudios semióticos en nuestro país, tal como muestra de manera fehaciente su impresionante Historia del pensamiento semiótico ${ }^{2}$; de la misma manera, fue también capaz de enfrentarse en el ámbito de los estudios peirceanos a complejas cuestiones filosóficas que iban más allá de la semiótica.

Organizaremos nuestra exposición en tres partes. En primer lugar, una breve semblanza personal de Wenceslao a partir de nuestro trato con él y de su relación con el Grupo de Estudios Peirceanos de la Universidad de Navarra. En segundo lugar, haremos referencia a los principales escritos de Wenceslao en relación con Charles S. Peirce y, por último, haremos una valoración general de su aportación.

\section{Breve semblanza personal de Wenceslao Castañares}

Wenceslao Castañares fue colaborador del Grupo de Estudios Peirceanos prácticamente desde sus inicios en 1994. Nuestro contacto con él comenzó en 1995 y le conocimos personalmente en 1996, cuando con poco más de un año de andadura el Grupo le invitaba a dar una conferencia en Pamplona. La buena conexión fue inmediata y nunca se interrumpió desde entonces. A lo largo de más de veinte años Wenceslao nos visitó en numerosas ocasiones, acudiendo siempre que se lo pedíamos a seminarios, jornadas lógicas y tribunales de tesis. Su ultimo mensaje, poco antes de morir, fue precisamente para hablarnos con ilusión de un nuevo proyecto que podíamos emprender juntos.

Nos gustaría destacar aquí las dos virtudes que a nuestro juicio sobresalen de manera particular en la vida académica de Wenceslao. Estas dos virtudes son la humildad y la laboriosidad. Ya su propia expresión verbal nos puede sonar algo así como "anticuada" o quizá "vintage", porque no están de moda ni cosechan "likes" en Instagram, pero son las virtudes realmente decisivas para una vida académica fecunda como ha sido la de Wenceslao.

Respecto de su sencilla humildad - la arrogancia es la enfermedad profesional de muchos filósofos y profesores en general - acudimos al riquísimo Diccionario de citas que preparó con José Luis González Quirós y nos emocionó comprobar que citaba allí en la voz "Humildad" aquellos dos pasajes de Teresa de Ávila en Las Moradas que tan bien cuadran a Wenceslao: "Pues mientras estamos en esta tierra, no hay cosa que más nos importe que la humildad" (Las Moradas, Moradas primeras,

Wenceslao Castañares, Historia del pensamiento semiótico, 1. La Antigüedad grecolatina, Trotta, Madrid, 2014; 2. La Edad media, Trotta, Madrid, 2018. 
2) y aquel otro: "Humildad, que es el ungüento de nuestras heridas" (Las Moradas, Moradas terceras, 2$)^{3}$. En esta cualidad suya se encierra muy probablemente la clave decisiva de su encanto, del atractivo personal de Wenceslao.

Wenceslao atendió siempre generosamente a todas las personas a las que le pedíamos que asesorara, y varios doctorandos terminaron considerándole como uno de sus maestros, pues les ayudó decisivamente en la redacción de su tesis doctoral. En las repetidas ocasiones en que sugerimos a alguien que acudiera a visitarle o que le escribiera para plantearle sus dudas y consultas sobre Peirce, siempre quedaba encantado porque se había sentido maravillosamente atendido, sin prisas, con atención cuidadosa. Sirva como testimonio lo que escribió Marta Susana López, desde Córdoba, Argentina:

He recibido la dolorosa noticia de la muerte de Wenceslao y quisiera hacerles llegar a todos los queridos colegas españoles mis condolencias. Él ha sido mi director de tesis de doctorado durante algunos años, tesis que yo no alcancé a terminar por problemas personales. Durante el tiempo que tuve el privilegio de relacionarme con él mediante correo electrónico, aprendí a valorarlo y estimarlo como persona, como científico y como docente. Reciban todos un fuerte abrazo ${ }^{4}$.

Merece la pena destacar esta virtud de la humildad por encima de todas precisamente porque no está de moda. Al considerar esto venía a nuestra memoria aquel dicho del famoso rabino Jacob Yitzakh al consolar a otro rabino poco afortunado que cuenta George Steiner en sus Lecciones de los maestros: "Vienen a mí porque me sorprende que vengan, y no van a ti porque a ti te sorprende que no vayan". "Aquí se encierra la clave de toda enseñanza por medio del ejemplo", concluye sabiamente Steiner ${ }^{5}$. La humildad se expresa en forma de amabilidad. Los profesores enseñamos más por lo que somos y por lo que hacemos que por lo que decimos. Y en esto Wenceslao ha sido un verdadero maestro.

La segunda cualidad de Wenceslao que queremos destacar es la de su laboriosidad, que se refleja en particular en su pulcritud y rigor académico. Copiamos por ejemplo dos preguntas suyas de un mensaje de diciembre del 2001:

¿Tienes documentada la definición medieval aliquid stat pro aliquo? Me refiero, naturalmente, a algún autor medieval. Tengo que escribir un artículo sobre el tema de la representación desde un punto de vista histórico y me gustaría aludir de forma precisa a esta cuestión". Y antes de esto decía: "Como casi siempre voy a pedirte algo, a condición de que no te suponga una "investigación" ad hoc. No me gustaría que te ocupara mucho tiempo.

Nuestro máximo experto en la noción de signo — que suele definirse como aliquid stat pro aliquo o expresiones parecidas - estaba en busca de la fuente original. La otra pregunta de aquel mensaje era: "En tu Taller de la filosofía aludes a la frase de Juan de Salisbury: ‘Enanos a hombros de gigantes' (Metal. III, 4). ¿Puedes darme la frase literal en latín? (Para el Diccionario)". Wenceslao empleaba la respuesta en

Wenceslao Castañares y José Luis González Quirós, Diccionario de citas, Noesis, Madrid, 2000 (2ª ed.), p. 568. Correo electrónico del 31 de octubre de 2018 a Izaskun Martínez, webmaster del Grupo de Estudios Peirceanos.

George Steiner, Lecciones de los Maestros, Siruela, Madrid, 2003, p. 148. 
la página inicial del volumen primero de Historia del pensamiento semiótico, en la que figura el famoso «quasi nanos gigantium humeris insidentes».

En resumen, pudimos comprobar muy bien a lo largo de los años la capacidad de trabajo de Wenceslao, su honestidad intelectual y su profunda humanidad.

\section{Principales escritos de Wenceslao Castañares en relación con C. S. Peirce}

Wenceslao Castañares fue el primero en realizar una tesis doctoral sobre Peirce en España. Su trabajo, titulado El signo: problemas semióticos y filosóficos, fue defendido en 1985 en la Universidad Complutense de Madrid. En ese trabajo, Wenceslao situaba a Peirce en el contexto de la historia de la semiótica, comparándolo acertadamente con otras tradiciones y teorías, como por ejemplo la de Saussure. Destaca especialmente la capacidad de Wenceslao de vislumbrar el futuro de los estudios sobre Charles S. Peirce, que apenas estaban empezando, y de hacerse una acertada idea de la proyección que tendría en el futuro el pensamiento peirceano, capaz de explicar no solo el signo en cuanto tal sino el conjunto de la razonabilidad y la acción humana. Como escribe en la introducción: "La semiótica que hasta ahora se ha desarrollado ha sido en gran medida una semiótica lingüística. Pero no debe limitarse a ese campo (...). Debe abrirse a otros ámbitos no específicamente lingüísticos como pueden ser los estéticos, los éticos, los científicos, etc.". Como escribió Fernando Zalamea en su exhaustivo catálogo razonado de la Bibliografía Peirceana Hispánica:

Se trata de la primera tesis doctoral hispánica dedicada en profundidad a Peirce. Son de gran importancia el esfuerzo de ordenamiento y síntesis, y el énfasis en el acordonamiento de las ideas de Peirce con la historia de la filosofía y de la semiótica. Algunos apartados son especialmente logrados y no serán superados en la bibliografía peirceana hispánica posterior ${ }^{6}$.

La tesis de Wenceslao fue años después publicada en la web del Grupo de Estudios Peirceanos. Como Wenceslao no se sentía muy orgulloso de algunas partes de aquel trabajo, tuvimos que superar su resistencia para escanear la tesis doctoral y publicarla en internet con una ilustrativa "Advertencia al lector: Veinte años después", que muestra bien la confusa situación de la semiótica de los años setenta y el trabajo clarificador que había supuesto para Wenceslao el estudio histórico de las fuentes textuales. Transcribimos a continuación algunas líneas de aquella "Advertencia", que ponen de manifiesto el carácter "peirceano" de Wenceslao:

Lo que más recuerdo de los momentos en que concebí el proyecto era la confusión en que parecía estar sumida la semiótica una vez desaparecida la euforia inicial con la que irrumpió en los ambientes intelectuales, especialmente franceses. Esta confusión tenía también un importante componente personal (a lo peor era más subjetivo que objetivo). De ahí que el propósito fundamental de la investigación fuera tratar de clarificar el panorama tal como yo lo contemplaba a finales de los años setenta. [...]

Fernando Zalamea, "Bibliografía Peirceana Hispánica", en Jaime Nubiola y Fernando Zalamea, Lo que C. S. Peirce dijo sobre España y lo que el mundo hispánico ha dicho sobre Peirce, Ediciones Universidad de Navarra, Pamplona, 2006, pp. 232-233. 
Creo que entre las cosas que siguen teniendo valor están esas calas realizadas en ciertos lugares estratégicos de la historia a la búsqueda de las teorías sobre la significación. [...] Espero que una versión mucho más amplia pueda ver la luz en un plazo razonable de tiempo. Del mismo modo, el análisis de las teorías de Saussure y Peirce quizá mereciera alguna revisión y, probablemente, una ampliación, pero me sigue pareciendo correcto. El conocimiento profundo de estos autores configuró, en no pocos aspectos, mi biografía intelectual. Esto es especialmente evidente en el caso de Peirce: no hay más que echar una mirada a mis publicaciones de los años ochenta y primeros noventa. Ese temor a comprometerme demasiado con el autor americano que el lector podrá apreciar en algunos pasajes de mi tesis terminó desapareciendo y no me produce rubor alguno confesar que soy hoy más peirceano que cuando defendí este trabajo ${ }^{7}$.

La tesis doctoral supuso para Wenceslao el inicio de una trayectoria de muchos años en la que, como él mismo afirmaba, sucumbió al "efecto Peirce". La lectura detenida y rigurosa del pensamiento de Peirce constituye, en palabras del propio Wenceslao, una "aventura intelectual con sentimientos encontrados que hacen de ella una experiencia nada fácil de olvidar"".

Entre las publicaciones peirceanas de Wenceslao es preciso destacar también su magnífico libro De la interpretación a la lectura de 1994, en el que aspira a aplicar las tesis semióticas en el novedoso campo de la comunicación. A partir de una impresionante erudición y de un conocimiento a fondo de muchos autores contemporáneos, apunta a:

la posibilidad de integrar las aportaciones realizadas desde teorías de la interpretación y la lectura en las investigaciones que es necesario llevar a cabo en el contexto de la comunicación de masas. Se aboga, pues, por la conveniencia de realizar una investigación de los procesos de recepción que, yendo más allá de los análisis de carácter cuantitativo, nos sitúe en el lugar en el que los destinatarios confieren sentido a los mensajes y poder vislumbrar así con mayores garantías qué efectos producen $^{9}$.

Este libro ha llegado a ser un manual de referencia para todo estudioso de la semiótica y de Peirce.

En la página web del Grupo de Estudios Peirceanos pueden consultarse varios artículos suyos que hemos recomendado en muchas ocasiones a quienes se inician en el estudio de Peirce, pues constituyen sin duda una de las fuentes de acceso más sólidas, rigurosas y claras a la semiótica y al pragmatismo de este pensador.

Recientemente hemos editado una compilación de los artículos de Wenceslao sobre Peirce en un volumen titulado Escritos sobre C. S. Peirce. Wenceslao Castañares (Ediciones Universidad de Navarra, 2019), que recoge quince textos escritos entre 1986 y 2008, los años en los que el estudio de Peirce se fue extendiendo deci-

Wenceslao Castañares, “Advertencia al lector: Veinte años después”, 2005, accesible online en $<\mathrm{http}$ ://www. unav.es/gep/RevisionCastanares.html>.

$8 \quad$ Wenceslao Castañares, "El efecto Peirce. Sugestiones para una teoría de la comunicación” (1996), Escritos sobre C. S. Peirce. S. Barrena y J. Nubiola (eds.), Ediciones Universidad de Navarra, 2019, p. 191.

9 Wenceslao Castañares, De la interpretación a la lectura, Iberediciones, Madrid, 1994, p. 19. 
sivamente en España. Los primeros escritos del volumen ponen de manifiesto cómo Wenceslao fue uno de los primeros en emprender en España el estudio de Peirce. En ese sentido supo abrir camino y explicar cosas que por entonces muy poca gente sabía. En ese volumen puede apreciarse con claridad cómo el conocimiento que tenía Wenceslao de Peirce va creciendo poco a poco a lo largo de los años. Hay en los textos algunas ideas recurrentes, pero, lejos de constituir meras repeticiones, se añade algo nuevo en cada vuelta y se van aportando nuevas luces. Lo que primero aparece solo en germen adquirirá toda su plenitud al retomar las ideas años después. En los capítulos finales encontramos textos como "La prueba y la probabilidad retórica", en el que realiza un acercamiento a la noción de prueba y lo probable a partir de Aristóteles y Peirce, o "El acto creativo: Continuidad, innovación y creación de hábitos", en el que trata de examinar con profundidad los fundamentos metafísicos de la noción de creatividad y sus aspectos lógico-semióticos. En esos textos finales es evidente el profundo dominio de la obra de Peirce que alcanzó Wenceslao, defendiendo una noción de lógica más amplia que, lejos de la racionalidad moderna y excluyente, supone un acercamiento más acertado a la humana manera de pensar combinada con la sensibilidad y la imaginación. El volumen en su conjunto es una buena muestra del crecimiento de la razonabilidad que Peirce defendía, y que Wenceslao logró de manera efectiva con su estudio y su constancia.

\section{La aportación de Wenceslao Castañares a los estudios peirceanos}

Como se ha visto, Wenceslao contribuyó decisivamente a dar a conocer la figura y el pensamiento de Peirce, especialmente en el ámbito hispanoamericano, y trató de fomentar siempre la cooperación académica y personal que Peirce propugnaba. A lo largo de su camino, Wenceslao supo enfrentarse con rigor a los aportes - y también a las insuficiencias - del pensamiento peirceano, y formó parte del diálogo que ese pensamiento permite entablar con diversas disciplinas y líneas de investigación.

Aunque Wenceslao llegó a Peirce a través de la semiótica, pronto fue consciente de la verdadera dimensión de sus teorías. Intuyó desde el principio que Peirce podía contribuir a superar muchas de las limitaciones del enfoque lingüístico y estructuralista — en la línea de Saussure — que reduce todo signo al lingüístico, y que olvida un enfoque más lógico y universal que Peirce puso de manifiesto con su concepción triádica del signo frente a la dualidad sujeto-objeto. Lejos de las modas, Peirce podía constituir un antídoto contra el "esnobismo semiótico", como afirma Wenceslao en "C. S. Peirce: historia de una marginación" $(1987)^{10}$.

Wenceslao supo por tanto reconocer el valor de la semiótica dentro del conjunto de la obra de Peirce, y defendió la necesidad de un estudio sistemático de su pensamiento para todo aspirante a semiótico. Así, afirmaba:

Para muchos, entre los que me encuentro, el acceso a la obra de Peirce ha tenido lugar a través de la semiótica. Pero enseguida es posible apreciar que, en primer lugar, no es ni la única ni quizá la principal puerta de acceso; en segundo, que, se entre por donde se entre, resulta necesario conocer, o al menos vislumbrar, los

\footnotetext{
Wenceslao Castañares, “C. S. Peirce: Historia de una marginación” (1987), Escritos sobre C. S. Peirce, p. 45.
} 
elementos sustentadores de una obra de la que se pueden desconocer en detalle algunas de sus partes, pero no su carácter arquitectónico ${ }^{11}$.

Wenceslao atravesó con valentía esa puerta de entrada, y su enfoque principalmente semiótico no fue impedimento para que se enfrentara a los aspectos más filosóficos de la obra de Peirce. Hay una sistematicidad en el pensamiento de Peirce que es necesario captar, como bien supo hacer Wenceslao a lo largo de los años, para comprenderlo y proseguirlo en una traducción libre: la única, como él mismo afirmaba, que nos permite avanzar en el conocimiento.

En este sentido, Wenceslao no se limita a reproducir servilmente unas posiciones históricas, sino que sobre todo — parafraseando a Hannah Arendt-aspira a comprender, a captar el sentido de la evolución de las ideas:

Había que rastrear las huellas que hicieran posible la reconstrucción de las fuentes históricas —explica en la "Advertencia al lector" del año 2005-. Una vez encontradas esas raíces que podían hacer más comprensibles sus teorías, esperaba llegar a tener algunas claves que me ayudaran a poner orden en el presente y otear los caminos por los que se podría caminar en el futuro ${ }^{12}$.

La originalidad y el vigor del pensamiento de Wenceslao son claros en sus escritos sobre Peirce. Wenceslao se enfrentó sin miedo a las teorías peirceanas, incluso a aquellas de honda raigambre metafísica: desde la nueva lista de categorías - primeridad, segundidad, terceridad-que vertebra su pensamiento y de modo especial su semiótica, pues el signo no podría entenderse sin la mediación característica de la terceridad, hasta teorías como el idealismo objetivo (la materia es "inteligencia desvirtuada"), o su cosmología de corte evolucionista. Se enfrenta en repetidas ocasiones a nociones peirceanas que es capaz de situar en un contexto mayor, tal como como hace por ejemplo con la abducción, esa peculiar operación lógica en la que se imbrican razón, imaginación e instinto, y que permite a Wenceslao llegar a una "racionalidad flexible" y en definitiva a explicar los aspectos más creativos de la persona humana. Su dominio del pensamiento peirceano le permitió, por tanto, abrir nuevos caminos a "soluciones imaginativas que, si no eliminan las diferencias, mantienen al menos la esperanza y la posibilidad del diálogo"13.

$* * *$

En conclusión, podemos considerar a Wenceslao, sin temor a equivocarnos, como uno de los primeros y más grandes estudiosos de Charles S. Peirce en España e Hispanoamérica. En el Grupo de Estudios Peirceanos hemos considerado siempre a Wenceslao como un maestro y un amigo, y quizá la mejor muestra de homenaje es tratar de seguir su luminoso ejemplo tanto en la humildad como en la laboriosidad.

11 Wenceslao Castañares, "El efecto Peirce. Sugestiones para una teoría de la comunicación” (1996), Escritos sobre C. S. Peirce, p. 192.

12 Wenceslao Castañares, "Advertencia al lector: Veinte años después”, 2005, accesible online en <http://www. unav.es/gep/RevisionCastanares.html>.

13 Wenceslao Castañares, “Abducción y traducción entre culturas” (1996), Escritos sobre C. S. Peirce, p. 186. 
Muchas personas de nuestro entorno han reconocido la pérdida de un gran investigador, pero sobre todo de una gran persona: su presencia perdurará entre nosotros y su legado ayudará sin duda a muchísimos estudiosos. 